\title{
Jean-Marc Ramos (dir.), La construction de l'idée de temps. Archives françaises (1876-1909)
}

Toulouse, Octarès Éditions, coll. « Témporalités », 2016, 406 p.

Jean-Bruno Renard

\section{OpenEdition}

Édition électronique

URL : http://journals.openedition.org/assr/28328

DOI : $10.4000 /$ assr.28328

ISSN : $1777-5825$

Éditeur

Éditions de l'EHESS

Édition imprimée

Date de publication : 31 décembre 2016

Pagination : 375

ISSN : 0335-5985

Référence électronique

Jean-Bruno Renard, « Jean-Marc Ramos (dir.), La construction de l'idée de temps. Archives françaises (1876-1909) », Archives de sciences sociales des religions [En ligne], 176 | octobre-décembre 2016, mis en ligne le 20 juillet 2017, consulté le 24 septembre 2020. URL : http://journals.openedition.org/assr/ 28328 ; DOI : https://doi.org/10.4000/assr.28328

Ce document a été généré automatiquement le 24 septembre 2020.

(c) Archives de sciences sociales des religions 


\title{
Jean-Marc Ramos (dir.), La construction de l'idée de temps. Archives françaises (1876-1909)
}

Toulouse, Octarès Éditions, coll. « Témporalités », 2016, 406 p.

\author{
Jean-Bruno Renard
}

\section{RÉFÉRENCE}

Jean-Marc Ramos (dir.), La construction de l'idée de temps.

1 Nombre de recherches menées aujourd'hui en sciences humaines prennent en compte la pluralité des temps sociaux. Les efforts déployés par les « temporalistes » français y sont pour beaucoup, notamment Jean-Marc Ramos, cheville ouvrière du bulletin de liaison Temporalistes (1984-2002), créé par William Grossin, puis de la revue Temporalités (depuis 2004), publications françaises qui n'ont rien à envier à l'incontournable Time \& Society anglo-saxon.

2 L'ouvrage a un double objectif : promouvoir les études sur le temps et répondre à une demande de textes fondateurs sur ce nouveau champ de recherche. Dans sa dense et belle introduction, «La construction de l'idée de temps» (p. 1-28), J.-M. Ramos montre comment, au tournant des $\mathrm{XIX}^{\mathrm{e}}$ et $\mathrm{xx}^{\mathrm{e}}$ siècles, des auteurs rompent avec la représentation de sens commun d'un temps unique, donné, pour faire émerger la conception savante d'un pluralisme temporel, soumis à la variété des cadres sociaux et des formes psychologiques. Toutefois, ces pionniers avancent en ordre dispersé, chacun présentant ses propres arguments, souvent liés à des disciplines et à des centres d'intérêt particuliers. Il faudra attendre la fin du $x^{e}$ siècle pour qu'apparaisse la préoccupation d'une théorie générale des « temporalités ».

3 Cette anthologie rassemble onze textes français rarement réédités, qui constituent des études pionnières sur le temps. Les amateurs d'archives apprécieront la reproduction 
en fac-similés de ces textes tirés de revues anciennes. Chacun d'eux est précédé d'une notice érudite, rédigée par un spécialiste, qui présente l'auteur.

4 Le texte de Grégoire Wyrouboff, «Remarques sur le calendrier de M. Comte » (1876), ouvre l'anthologie. C'est une critique sévère, au nom même du positivisme comtien, du projet de calendrier d'Auguste Comte, auquel l'historien des sciences reproche son incomplétude et ses préjugés dans le choix des grands hommes qui ont contribué au progrès de l'humanité.

5 Le texte du très monarchiste baron Edmond de Rivières, "Inscription et devises horaires » (1881), est un précieux inventaire des devises figurant sur des cadrans solaires. Ces brèves inscriptions révèlent la variété des rapports au temps : évocation astronomique de la course du soleil, rappel de la brièveté de la vie, incitation au travail ou à la prière, invitation à profiter du moment présent... On comprend tout l'intérêt qu'il y a pour des chercheurs à se pencher sur ces " proverbes temporalisés », comme l'ont fait Max Reinert et J.-M. Ramos en analysant un important corpus de devises de cadrans solaires, montrant ainsi le passage d'une conception ancienne théocentrée à une conception égocentrée (Temporalités, $\left.\mathrm{n}^{\circ} 1,2004, \mathrm{p} .19-36\right)$.

6 Le linguiste Paul Regnaud a écrit un texte bref, mais fondateur pour l'étude du vocabulaire du temps: «L'idée de temps. Origine des principales expressions qui s'y rapportent dans les langues indo-européennes" (1885). L'auteur montre que, dans cette famille linguistique, le lexique du temps dérive le plus souvent de termes désignant soit la lumière et la chaleur, soit la vigueur, la vie et la durée de la vie.

7 Philosophe oublié que l'on redécouvre aujourd'hui, Jean-Marie Guyau a publié en 1885 un texte sur "L'évolution de l'idée de temps dans la conscience", anticipant une phénoménologie de l'expérience du temps.

8 Rimbaldien distingué, J.-M. Ramos n'a pas résisté à la tentation d'insérer dans son anthologie le célèbre poème "Barbare", écrit par l'auteur des Illuminations (1886). L'anthologiste se justifie en montrant de manière convaincante qu'Arthur Rimbaud évoque ici un temps autre que celui qui passe: le temps du rêve, le temps de la " cinquième saison ", que les psychanalystes identifieront au temps de l'inconscient.

9 Avec «Les mines et les huit heures» (1902), le sociologue et économiste Paul de Rousiers, qui appartient à l'école sociographique de Le Play, donne son analyse d'un projet de loi sur la réduction à huit heures de la journée de travail dans les mines de houille. Précurseur de la mise en garde contre les « effets pervers» (Raymond Boudon), de Rousiers redoute que la loi provoque mécaniquement une baisse de la production, qui nuira finalement au monde ouvrier. Il plaide pour que la réforme du temps de travail s'accompagne de mesures de modernisation pour améliorer l'exploitation minière. Une réflexion sur les temps de travail, de transport, de repos, de loisir et d'instruction est au cœur de ce texte qui, même après la disparition des mines, reste d'actualité !

10 Une anthologie sur le temps se devait d'y faire figurer Henri Bergson. Le texte présenté ici est rare : il s'agit du résumé par un anonyme du cours donné au début des années 1900 par Bergson au Collège de France sur "L'idée de temps", publié en 1902 dans la Revue de philosophie. On y trouve l'affirmation qu'« il n'y a pas une forme seulement de la durée comme il n'y a qu'une forme de l'espace, mais bien autant d'espèces de durées qu'il y a d'espèces d'êtres qui durent ». 
11 Injustement oublié au profit de Marcel Mauss, avec lequel il publia dans l'Année sociologique les célèbres essais sur le Sacrifice et sur la Magie, Henri Hubert est l'auteur d'une "Étude sommaire de la représentation du temps dans la religion et la magie » (1905). Les rites ont toujours un " minimum de détermination temporelle ", c'est-à-dire qu'il y a un moment fixé pour les accomplir. Les calendriers liturgiques, les almanachs de jours fastes et néfastes, les rituels magiques font plus que s'inscrire dans le temps, ils créent le temps. Et Hubert conclut que c'est par abstraction que l'on est passé d'un temps qualitatif, hétérogène, circulaire, à la notion moderne d'un temps quantitatif, homogène et linéaire.

Benjamin Bourdon fait entrer l'étude du temps en psychologie expérimentale par son texte «La perception du temps » (1907), qui est en réalité la perception de la durée, façonnée le plus souvent rétrospectivement par la mémoire. La même année, Gabriel Revault d'Allonnes publie son ouvrage Les inclinations. Leur rôle dans la psychologie des sentiments, dans lequel apparait pour la première fois l'affirmation d'une "psychologie du temps» dans un chapitre, reproduit dans l'anthologie, intitulé «Dissociation du sentiment de temps ». Le psychologue y expose des cas de désorientations temporelles, qui le conduisent à distinguer trois registres différents: «le temps sensoriel», «le temps affectif» et «le temps intellectuel». Ces deux textes sont fondateurs d'une psychologie du temps qui sera développée plus tard, notamment par Paul Fraisse dans les années 1950.

L'anthologie se termine par un texte bien connu des lecteurs des Archives de Sciences Sociales des Religions, "Sociologie religieuse et théorie de la connaissance" (1909), publié par Émile Durkheim dans la Revue de métaphysique et morale (t. XVII, $\left.\mathrm{n}^{\circ} 6\right)$ et qui constituera l'Introduction des Formes élémentaires de la vie religieuse (1912). Durkheim y résume sa conception sociologique de la religion. Non seulement celle-ci est « une chose éminemment sociale ", mais elle est aussi à l'origine de nos catégories de pensée : l'espace, le genre, le nombre, la cause, la substance... et bien sûr le temps : les " points de repères indispensables, par rapport auxquels toutes choses sont classées temporellement, sont empruntés à la vie sociale. [...] Toute coopération suppose une notion commune du temps qui permette aux hommes de concerter leurs rencontres et ceux de leurs actes qui doivent être accomplis en commun ». Durkheim reconnaît au passage sa dette envers Henri Hubert sur cette question.

Destinée aux chercheurs en sciences humaines, cette anthologie est particulièrement recommandée à ceux qui mènent des travaux en sciences sociales des religions. Du texte sur le calendrier de la religion positiviste jusqu'à ceux de Hubert et Durkheim, en passant par la mise en évidence de la dimension religieuse des devises des cadrans solaires, cette anthologie confirme l'idée que la notion de temps est née de la pratique religieuse. On comprend alors pourquoi François-André Isambert a vu en la personne d'Henri Hubert, historien des religions, le fondateur de la "sociologie du temps" («Henri Hubert et la sociologie du temps", Revue française de sociologie, vol. XX, $n^{\circ} 1$, 1979, p. 183-204). 